\title{
Low Frequency (30-110 MHz) Radio Imaging Observations Of Solar Coronal Mass Ejections
}

\author{
R. Ramesh \\ Centre for Research and Education in Science and Technology, Indian Institute of \\ Astrophysics, Hosakote 562 114, Bangalore, INDIA \\ email: ramesh@iiap.res.in
}

\begin{abstract}
Ground based radio imaging observations play an useful role in the study of mass ejections from the solar corona since they do not have the limitation of an occulter and both the disk/limb events can be detected early in their development, particularly via the thermal bremmstrahlung emission from the frontal loop of the CME. I present here some of the recent results on the above topic using data obtained with the Gauribidanur radioheliograph, near Bangalore in India.
\end{abstract}

Keywords. Sun: general, Sun: corona, Sun: coronal mass ejections (CMEs), Sun: radio radiation

\section{Introduction}

Studies of the influence of solar activity on our terrestrial environment has taken on increasing importance in recent years, as the realization of just how damaging space influences can be. The state of near-Earth space environment is significantly controlled by CMEs, the most-geoeffective manifestation of solar activity (Gosling et al. 1991). CMEs are large-scale magneto-plasma structures that erupt from the Sun and propagate through the interplanetary medium with speeds ranging from only a few $\mathrm{km} \mathrm{s}^{-1}$ to nearly $3000 \mathrm{~km} \mathrm{~s}^{-1}$. They carry typically $10^{15} \mathrm{gm}$ of coronal material. Observations from instruments such as the Large Angle and Spectroscopic Coronagraph (LASCO: Brueckner et al. 1995) onboard the Solar and Heliospheric Observatory (SOHO: Fleck et al. 1995) have now revolutionized our perception and understanding of the solar eruptive events. But, by their very nature, the coronagraphs have an occulting disk to block the direct photospheric light and so the early life/kinematics of a CME in the low corona cannot be studied using them. One needs non-coronagraphic data to obtain information on the early evolution of CMEs, in particular for those directed along the Sun-Earth axis which occur far from the plane of the sky. The latter originate on the visible hemisphere of the Sun and appear as a 'halo' of expanding, circular brightening that completely surrounds or spans a large angle outside the occulting disk of a coronagraph (Howard et al. 1982). The Earthward-moving events are geophysically important, in the context of space-weather related phenomena such as geomagnetic storms. Measurements of CME properties in the lower corona are significant for several reasons. Foremost among them is the general assumption that CMEs have a constant speed behind the occulting disk of a coronagraph. This has often caused controversial results while comparing the CME onset with other solar activity signatures (see Ramesh \& Ebenezer 2001b; Ramesh \& ShanmughaSundaram 2001c for possible radio signatures in the solar corona prior to as well as during the lift-off time of CMEs). The kinematic evolution of a CME can be described in a three-phase scenario: the initiation phase, impulsive acceleration phase, and propagation phase. Among 

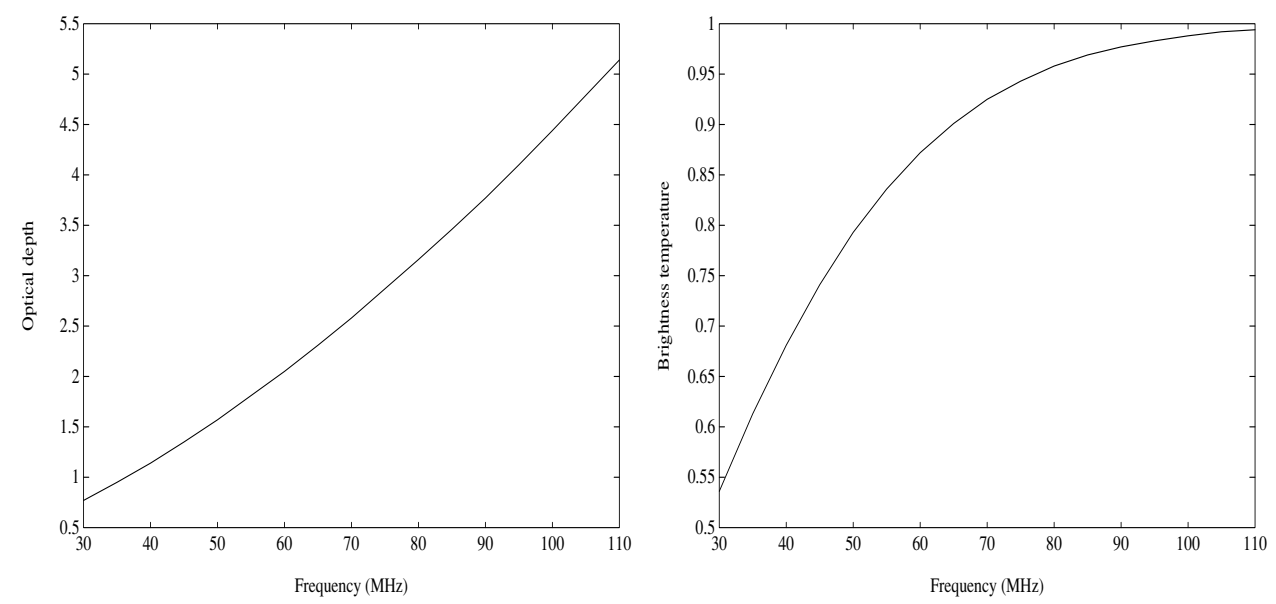

Figure 1. Variation of optical depth and observed brightness temperature in the solar corona as a function of plasma frequency. The electron density model of Newkirk (1961) and a coronal electron temperature $\left(T_{e}\right)$ of $\sim 10^{6} \mathrm{~K}$ was used for the calculations. The frequency range in the above Figure corresponds to a height range of $\sim 1.2-2.0 \mathrm{R}_{\odot}\left(\mathrm{R}_{\odot}=\right.$ solar radius $\left.=6.96 \times 10^{5} \mathrm{~km}\right)$, from the center of the Sun.

these, the first 2 phases take place primarily in the low corona. These suggest that a more complete description of the motion of a CME, close to the Sun, is crucial for a better prediction of its characteristics at higher altitudes. Again, what one measures usually from a time-lapse movie of the images obtained with a coronagraph is the speed at which a CME spreads in the plane of the sky. This will at best be only a lower limit to the true value (i.e. the speed in the three-dimensional space) especially in the case of an Earth-directed 'halo' CME as it will lie away from the plane of the sky. In this respect, imaging observations particularly at frequencies $\leqslant 110 \mathrm{MHz}$ play an important role since the radio instruments do not have the limitation of an occulting disk and a CME can be detected early in its development via the thermal bremmstrahlung radiation from its frontal loop (Sheridan et al. 1978; Gopalswamy \& Kundu 1992; Kathiravan, Ramesh \& Subramanian 2002). The frontal structure of a CME has a large optical depth in the above frequency range, and can be readily observed (Bastian \& Gary 1997; Gopalswamy 1999; Ramesh, Kathiravan \& Sastry 2003). Note that the optical depth of the background corona is not so large in the range $30-110 \mathrm{MHz}$ (left hand side of Figure 1). Due to this, the observed brightness temperature is generally less than the electron temperature of the corona (right hand side of Figure 1). Therefore the presence of a weak density enhancement like a CME in the solar atmosphere can be noticed with comparitively better contrast. Also one can observe activity at any longitude similar to X-ray and EUV wavelengths (Ramesh 2000a). In addition to direct imaging, ray tracing analysis of the thermal radio counterpart of a CME also play a vital role since one can localize the position of the associated density enhancement in a three-dimensional space (Ramesh \& Sastry 2000b; Kathiravan, Ramesh \& Subramanian 2002; Kathiravan \& Ramesh 2004). One can also use imaging observations of the apparent angular broadening of a distant cosmic source when the radio waves from it undergo scattering while happen to pass through a CME, to infer characteristics of the latter particulary at large distances from the Sun (Ramesh, Kathiravan \& Sastry 2001a). 

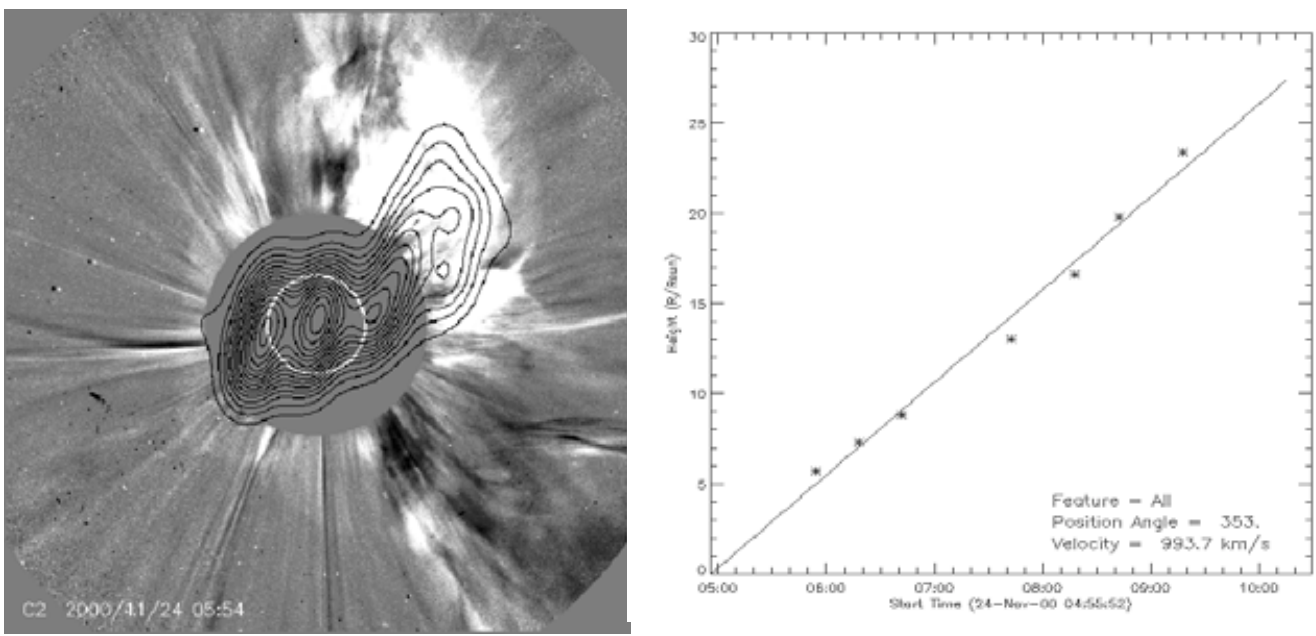

Figure 2. LEFT - A composite of the LASCO C2 difference image (05:54-05:06 UT) and the Gauribidanur radioheliogram (05:25 UT) of the CME event observed on November 24, 2000. The inner circle indicates the solar limb, and outer circle is the occulting disk of the coronagraph. It extends approximately upto $2.2 \mathrm{R}_{\odot}$ from the center of the Sun. Solar north is straight up and east is to the left. The CME can be clearly noticed as a bright structure above the north-west quadrant of the occulting disk in the coronagraph image and as a well-defined bulging of the contours at the corresponding location in the radioheliogram. RIGHT - Height-time plot of the whitelight $\mathrm{CME}$ obtained using LASCO C2/C3 measurements. Note that the first data point for the plot was available only at a height of about $5.71 \mathrm{R}_{\odot}$ from the center of the Sun.

\section{Case studies}

\subsection{The event of November 24, 2000}

The radio data reported were obtained at $109 \mathrm{MHz}$ with the Gauribidanur radioheliograph (GRH) operating near Bangalore in India (Ramesh et al. 1998a; Ramesh 1998b; Ramesh, Subramanian \& Sastry 1999). Note that after the closing down of the Culgoora and Clark Lake radioheliographs, the GRH is the only instrument that is presently in operation for radio observations of the solar corona in the frequency range 30$110 \mathrm{MHz}$. The latter corresponds to an altitude range of $\sim 1.2-2 \mathrm{R}_{\odot}$ (from the center of the Sun) in the solar atmosphere. According to the CME list for the year 2000 (http://cdaw.gsfc.nasa.gov/CME_list), the LASCO C2 coronagraph onboard SOHO observed a full 'halo' CME on November 24, 2000 around 05:30 UT, the time at which it was first noticed in its field of view. The left half of Figure 2 shows a composite of the LASCO $\mathrm{C} 2$ difference image of the event at 05:54 UT obtained by subtracting a pre-event image at 05:06 UT, and the radioheliogram obtained with the GRH at 05:25 UT. The CME can be noticed as a bright feature above the north-west quadrant of the occulting disk of the coronagraph. The contours in the radioheliogram also show a well-defined bulging at the corresponding location. The extrapolated lift-off time of the CME was 04:55:52 UT. Its estimated linear speed in the plane of the sky was $994 \mathrm{~km} / \mathrm{s}$ (right half of Figure 2). A quadratic fit to the height-time data gave an acceleration of $72 \mathrm{~m} / \mathrm{s}^{2}$ for the event. Figure 3 shows the radioheliogram obtained with the GRH at 04:55, 05:05, 05:15 \& 05:25 UT on that day. In addition to the discrete sources on the disk, one can clearly observe enhanced radio emission in close spatial correspondence with the whitelight CME described above, at all the above 4 epochs. Its estimated peak brightness temperature $\left(T_{b}\right)$ was found to be $\sim 10^{5} \mathrm{~K}$. A comparison of the LASCO and GRH images clearly indicates that the radio enhancement moved in the same direction as the whitelight CME. Also their 

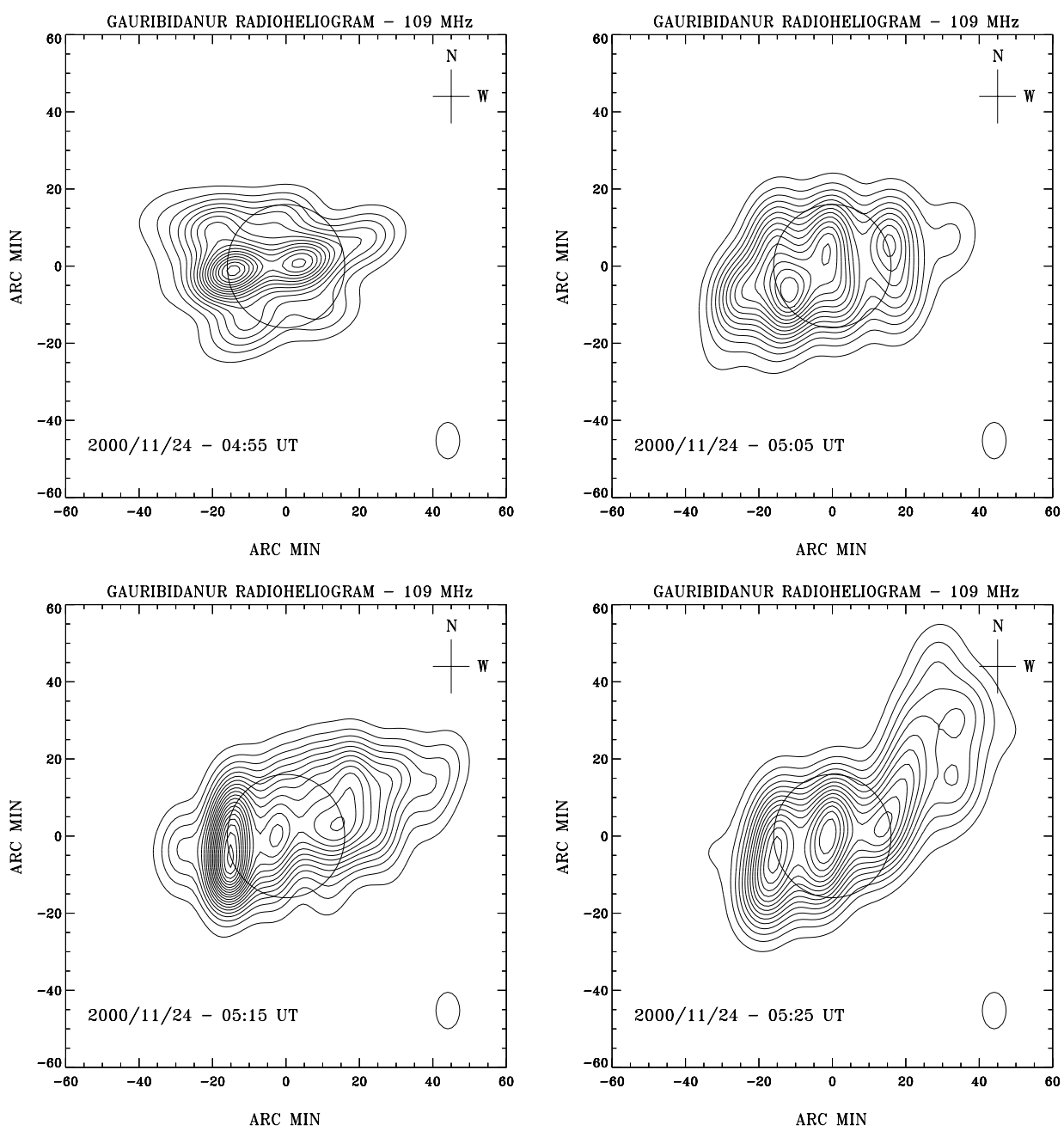

Figure 3. Radioheliogram obtained with the GRH on November 24, 2000 at different epochs. The open circle at the center is the solar limb. The instrument beam is shown near the bottom right corner. 04:55 UT - The peak $T_{b}$ is $\approx 4.18 \times 10^{6} \mathrm{~K}$, and the contour interval is $0.27 \times 10^{6} \mathrm{~K}$. 05:05 UT - The peak $T_{b}$ is $\approx 6.08 \times 10^{6} \mathrm{~K}$, and the contour interval is $0.34 \times 10^{6} \mathrm{~K}$. 05:15 UT - The peak $T_{b}$ is $\approx 6.1 \times 10^{6} \mathrm{~K}$, and the contour interval is $0.39 \times 10^{6} \mathrm{~K}$. 05:25 UT - The peak $T_{b}$ is $\approx 5.6 \times 10^{6} \mathrm{~K}$, and the contour interval is $0.29 \times 10^{6} \mathrm{~K}$.

appearances are closely similar. We therefore conclude that the former is the radio counterpart of the latter. We estimated the velocity of the 'radio CME' from the displacement of its centroid, and the values are $1087 \pm 73 \mathrm{~km} \mathrm{~s}^{-1}$ and $1185 \pm 73 \mathrm{~km} \mathrm{~s}^{-1}$ during the interval 05:05-05:15 UT and 05:15-05:25 UT, respectively. This gives it an effective acceleration of $\sim 157 \pm 121 \mathrm{~m} \mathrm{~s}^{-2}$. Note that this is about a factor of 2 more than the acceleration of the whitelight CME obtained using LASCO C2/C3 measurements whose first point was available only at a height of about $5.71 \mathrm{R}_{\odot}$ from the center of the Sun.

\subsubsection{Analysis and Results}

Single frequency observations of CME associated discrete radio sources travelling outwards to large heights $\left(\sim 2-3 \mathrm{R}_{\odot}\right)$ in the solar corona are generally attributed to non-thermal continuum emission from moving type IV radio bursts [see Dulk (1980) and 
Table 1. Characteristics of 'radio CME'

\begin{tabular}{ccccccc}
\hline Time & $\begin{array}{c}\text { Co-ordinates } \\
\text { of the } \\
\text { centroid }\end{array}$ & $\begin{array}{c}\text { Position } \\
\text { angle }\end{array}$ & $\begin{array}{c}\text { Brightness } \\
\text { temperature } \\
\left({ }^{\circ} \mathrm{K}\right)\end{array}$ & $\begin{array}{c}\text { Electron } \\
\text { density } \\
\left(\mathrm{cm}^{-3}\right)\end{array}$ & $\begin{array}{c}\text { Mass } \\
(\mathrm{g})\end{array}$ & $\begin{array}{c}\text { Magnetic } \\
\text { field } \\
(\mathrm{G})\end{array}$ \\
\hline 05:05 UT & $0.22 \mathrm{~N}, 1.06 \mathrm{~W}$ & $282^{\circ}$ & $3.34 \times 10^{5}$ & $1.24 \times 10^{8}$ & $2.68 \times 10^{16}$ & 2.40 \\
05:15 UT & $1.09 \mathrm{~N}, 1.41 \mathrm{~W}$ & $308^{\circ}$ & $2.86 \times 10^{5}$ & $4.46 \times 10^{7}$ & $3.82 \times 10^{16}$ & 1.33 \\
05:25 UT & $2.03 \mathrm{~N}, 1.81 \mathrm{~W}$ & $318^{\circ}$ & $1.92 \times 10^{5}$ & $2.81 \times 10^{7}$ & $4.42 \times 10^{16}$ & 0.86 \\
\hline
\end{tabular}

the references therein], since the observed $T_{b} \geqslant 10^{7} \mathrm{~K}$, is usually higher than that due to the emission from the background 'quiet' Sun $\left(\sim 10^{6} \mathrm{~K}\right)$ which is purely thermal in nature. But in the present case, the $T_{b}$ of the enhanced radio emission observed at the location of the whitelight CME is less than the electron temperature $\left(T_{e}\right)$ of the solar corona $\left(\approx 1.4 \times 10^{6} \mathrm{~K}\right.$, Fludra et al. 1999$)$. Also no type IV emission was reported during our observing period on November 24, 2000 (Solar Geophysical Data, January 2001). We would like to point here that optically thin synchrotron radiation from the non-thermal electrons entrained in the magnetic field of the CME could also give rise to low values of $T_{b}\left(\sim 10^{4}-10^{5} \mathrm{~K}\right)$ as shown recently by Bastian et al. (2001) for the event of April 20,1998 . But we could not verify the above (through spectral index estimation) in the present case, since the radio imaging data is available at only one frequency. However it is to be noted that the CME event described here was not accompanied by any nonthermal continuum emission in the metric range unlike the event reported by Bastian et al. (2001) [see Solar Geophysical Data, June 1998 for details]. Therefore it is possible that the CME associated enhanced radio emission observed by us in the present case is most likely thermal in nature, and the excess emission observed off the limb in the north-west quadrant of the GRH images is due to bremmstrahlung from the extra electrons associated with the 'halo CME'. Its mass $(M)$ is given by (Sheridan et al. 1978, Gopalswamy \& Kundu 1992),

$$
M=2 \times 10^{-24}\left[5 f^{2} T_{e}^{1 / 2} T_{b} L^{-1}\right]^{1 / 2} V \quad \mathrm{~g}
$$

where $f(\mathrm{MHz})$ is the observing frequency and $L\left(\mathrm{R}_{\odot}\right)$ is the depth of the radio enhancement along the line of sight. The latter is unknown and is taken to be the same as the observed radial width. The volume $(V)$ of the region of enhanced emission was determined by multiplying its radial and lateral width with the depth along the line of sight. We assumed that the coronal plasma is a fully ionized gas of normal solar composition ( $90 \%$ hydrogen and $10 \%$ helium by number), and each electron is associated with approximately $2 \times 10^{-24} \mathrm{~g}$ of material. In addition to the above, we also derived the magnetic field strength $(B)$ associated with the density enhancement assuming that the plasma $\beta \approx 0.05$, as found by Vourlidas et al. (2000) for some of the LASCO CMEs at about the same height range as the 'radio CME' described here. Table 1 lists the values of the different parameters of the latter obtained using the difference images with respect to that at 04:55 UT. The distance $(s)$ travelled by a CME in a given time interval $(t)$ can be found if its initial speed $(u)$ and acceleration $(a)$ are known. For the values of $u$ $=1087 \mathrm{~km} / \mathrm{s}, a=157 \mathrm{~m} / \mathrm{s}^{2}$ and $t=29 \mathrm{~min}$ [time difference between the last and first height measurement using GRH (05:25 UT) and LASCO data (05:54 UT), respectively], we found that the centroid of the 'radio CME' should be located at a height of $5.78 \mathrm{R}_{\odot}$ from the center of the Sun, at 05:54 UT. According to the LASCO measurements, the leading edge of the CME was located at a height of 5.71 $\mathrm{R}_{\odot}$ at 05:54 UT. 
R. Ramesh

\subsection{The event of June 2, 1998}

There is no direct measurement of the parameters/kinematics of a CME along the line of sight. For calculations like the volume of a CME, one generally assumes that the depth of the CME along the line of sight is the same as its radial width. We present here a method using which one can estimate the parameters of a CME separately along each direction in a three-dimensional space. According to the CME catalog for the year 1998, the LASCO C2 coronagraph observed a massive CME on June 2, 1998 around 08:08 UT (the time at which it was first noticed in its field of view) The above CME event was also accompanied by an exceptionally bright prominence eruption. The left hand side of Figure 4 shows a composite of the radioheliogram obtained with the GRH at $109 \mathrm{MHz}$ around 07:30 UT and the LASCO C2 image of the prominence eruption/CME obtained at 10:29:34 UT, on June 2, 1998. The leading edge of the CME was observed at a projected height of $2 \mathrm{R}_{\odot}$ around 07:40 UT in the field of view of the LASCO C1 coronagraph (Plunkett et al. 2000). This suggests that the extended, faint radio emission at the corresponding location in the composite picture on the left hand side of Figure 4 might be the counterpart of the former. The other bright whitelight feature in the southeast quadrant is probably associated with the CME that took place around 03:00 UT at a position angle (PA, measured counter clockwise from solar north) of $\approx 118^{\circ}$, on that day. One can clearly notice its radio counterpart also at the corresponding location. The peak brightness temperature of the above two radio counterpart of the whitelight CME were $\sim 10^{5} \mathrm{~K}$. This indicates that the emission is most likely thermal in nature. Also no nonthermal emission was reported during our observing period (Solar Geophysical Data, August 1998).

\subsubsection{Ray tracing method}

The brightness distribution of the 'quiet' Sun was computed theoretically from centimeter to meter wavelengths for the first time by Smerd (1950). He assumed a spherically symmetric corona to derive the solution by numerical integration of the radiative transfer equation for an ionized medium. The existence of density enhancements make the corona asymmetric and one has to take recourse in a more involved ray tracing technique to derive the brightness distribution. Such calculations were carried out by Newkirk (1961) and he derived the brightness profiles at short wavelengths. Sastry, Shevgaonkar \& Ramanuja (1983) used a ray tracing technique similar to that of the latter to explain the one-dimensional brightness distribution observed by them in the decameter wavelength range. We have now extended the above scheme to the analysis of an observed two-dimensional thermal radio brightness distribution. In order to simulate the observed radio brightness distribution superposed on the LASCO C2 image in the left hand side of Figure 4, we calculated the brightness distribution of the solar corona using various types of electron density and temperature distributions. Though in principle one could use any standard electron density distribution model for the solar corona in which the rays are to be traced, we describe our technique using a model similar to that of Newkirk (1961). The electron density at any point in the corona is given by,

$$
N_{e}(\rho)=N_{0}\left[1+C_{n} \exp \left(-\beta^{2}\right)\right] \mathrm{cm}^{-3}
$$

where $N_{0}=4.2 \times 10^{4.32 / \rho}$ (the spherically symmetric component of the background 'quiet' Sun) and $\rho$ is the radial distance from the center of the Sun. The constant $C_{n}$ is the strength of the density enhancement/depletion, and $\beta$ depends on its location and 

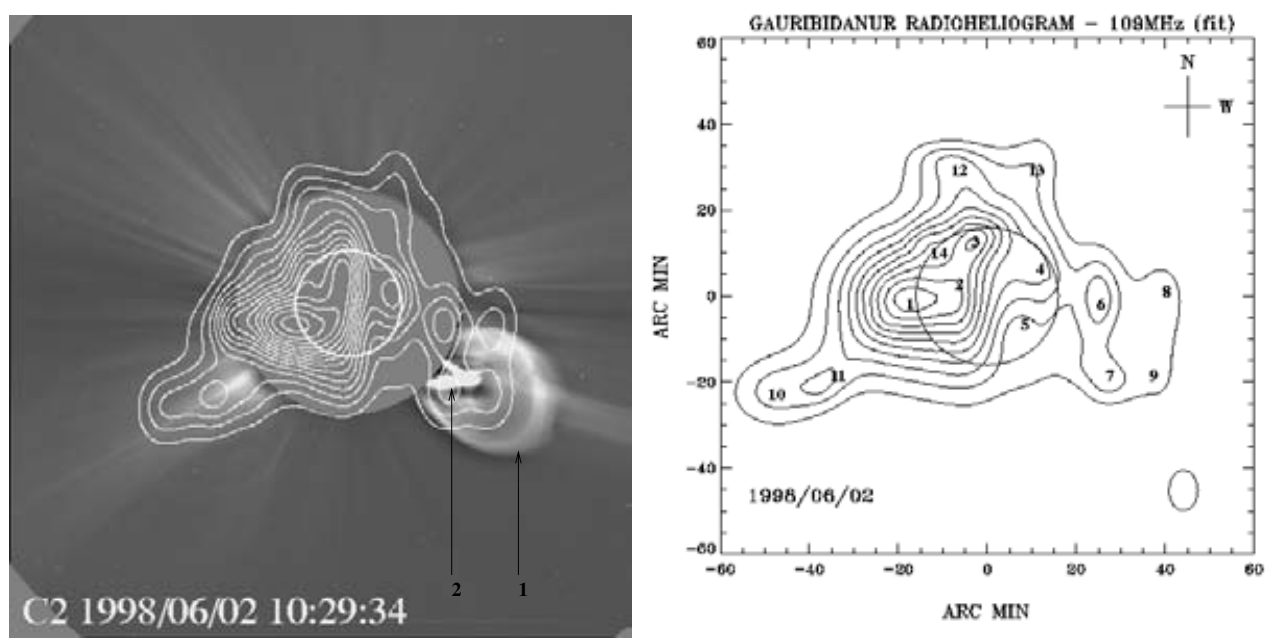

Figure 4. LEFT - A composite of the radioheliogram obtained with the GRH at $109 \mathrm{MHz}$ around 07:30 UT and LASCO C2 image of the giant prominence eruption/CME at 10:29:34 UT on June 2, 1998. The peak brightness temperature in the radio map is $\sim 1.3 \times 10^{6} \mathrm{~K}$. The contours are in interval of $8.6 \times 10^{4} \mathrm{~K}$. The arrows labelled $1 \& 2$ indicate the CME and the prominence, respectively. RIGHT - Radio brightness distribution of the Sun at $109 \mathrm{MHz}$ obtained through ray tracing calculations. The peak brightness temperature is $\sim 1.25 \times 10^{6} \mathrm{~K}$. The contours are in interval of $10^{5} \mathrm{~K}$. The numbers 1-14 indicate the location of the centroid of the discrete sources used in the ray tracing calculations. The sources 6-9 correspond to the observed enhancement at the location of the CME in the south-west quadrant.

size. It is given by,

$$
\beta^{2}=\frac{\left(x-x_{o}\right)^{2}}{2 \sigma_{x}^{2}}+\frac{\left(y-y_{o}\right)^{2}}{2 \sigma_{y}^{2}}+\frac{\left(z-z_{o}\right)^{2}}{2 \sigma_{z}^{2}}
$$

where $\sigma_{x}, \sigma_{y}, \sigma_{z}$ and $x_{o}, y_{o}, z_{o}$ are the size (along the respective axes) and the location of the centroid of the density enhancement/depletion. Here $x$ is towards the Earth (along the line of sight), and the $x y$-plane contains the axis of the localised region. $y \& z$ represent the longitudinal and lattitudinal directions on the Sun. All distances are in units of $\mathrm{R}_{\odot}$. For the localised regions, we used a model in which the density falls off as a Gaussian function along the $x, y \& z$ directions, from their centroid. To determine the brightness temperature $\left(T_{b}\right)$ at some point in the solar corona, rays initially directed towards that point are traced [using the technique described in Newkirk (1961)] from the Earth towards the Sun until the optical depth $(\tau)$ reaches a large value or the ray is moving away from the Sun and is atleast $5 \mathrm{R}_{\odot}$ from the Sun. The brightness temperature $\left(T_{b}\right)$ for any ray path is evaluated using the following integral,

$$
T_{b}=\int_{0}^{\tau} T_{e} e^{-\tau} d \tau
$$

where $T_{e}$ is the coronal electron temperature. The above procedure is repeated for different values of $y \& z$, and the results are stored in a two-dimensional array. After trial and error, we were able to reproduce the observed brightness distribution by assuming a background corona of uniform brightness temperature $\left(T_{e}=1.4 \times 10^{6} \mathrm{~K}\right)$ and a density profile equal to 0.35 times that given by equation (2.2). These values agree well with that reported recently by Fludra et al. (1999) for the 'quiet' Sun. The right hand side map in Figure 4 shows the brightness distribution obtained using the ray tracing technique described above. There is a good correspondence with the observed radio brightness 

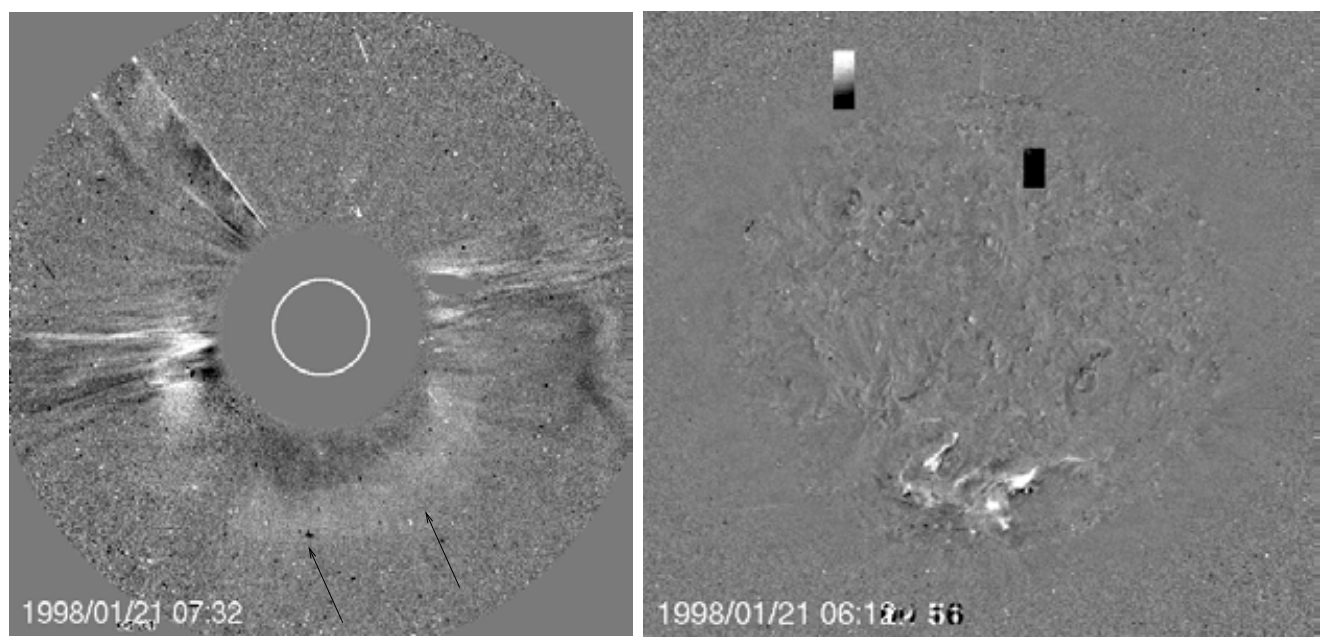

Figure 5. LEFT - Difference image (07:32-06:06 UT) of the 'halo' CME event observed with the LASCO C2 coronagraph on January 21, 1998. The CME can be noticed as a faint ring (indicated by the arrow marks) above the occulter of the coronagraph in the southern quadrant. RIGHT - A running difference image of the southern polar crown filament eruption of January 21, 1998 obtained at 06:10 UT with the Extreme ultra-violet Imaging Telescope (EIT) onboard SOHO at $195 \AA$.

distribution on the left hand side in Figure 4. The numbers 1-14 indicate the location of the centroid of the discrete sources used in our ray tracing calculations [see Kathiravan, Ramesh \& Subramanian 2002 for details] It is to be noted here that we have not included scattering (by small scale density inhomogeneities in the solar corona) since their effects are more pronounced mainly at decameter wavelengths (Aubier, Leblanc \& Boischot 1971). The sources 6-9 correspond to the observed enhancement at the location of the CME in the south-west quadrant in Figure 4 (left hand side) and their average electron density is $\approx 2.65 \times 10^{7} \mathrm{~cm}^{-3}$. This is about 17 times greater than the ambient density at $2.8 \mathrm{R}_{\odot}$, the mean radial distance of the above sources. A knowledge of the width of the structure along the line of sight enabled us to calculate its volume and hence the mass in a straightforward manner. The estimated value of the mass is $2.02 \times 10^{15} \mathrm{~g}$.

\subsection{The event of January 21, 1998}

One of the results that came out from our ray tracing calculations described in section 2.2.1 was the possibility to obtain the location of the various discrete sources along the line of sight direction also, in a straight forward manner. This provides a technique to determine the three-dimensional position co-ordinates of the thermal radio counterpart of a density enhancement in the corona at a given epoch and estimate its space speed under situations where it shows noticeable displacement as a function of time. It is obvious that if the observed brightness distribution evolves with time, then the different parameters used in the ray tracing calculations will be unique to the radio map obtained at a particular epoch. According to the CME catalog for the year 1998, a full 'halo' event was observed on January 21, 1998. The event was first observed above the southern quadrant of the occulting disk of the LASCO C2 coronagraph around 06:37:25 UT. Its leading edge was located at a height of about $2.75 \mathrm{R}_{\odot}$ at that time. The left half of Figure 5 shows a difference image of the event obtained at 07:32 UT by subtracting a pre-event image taken at 06:06 UT. One can notice the CME as a faint ring like feature in the southern hemisphere. The estimated linear speed of the leading edge of the CME 

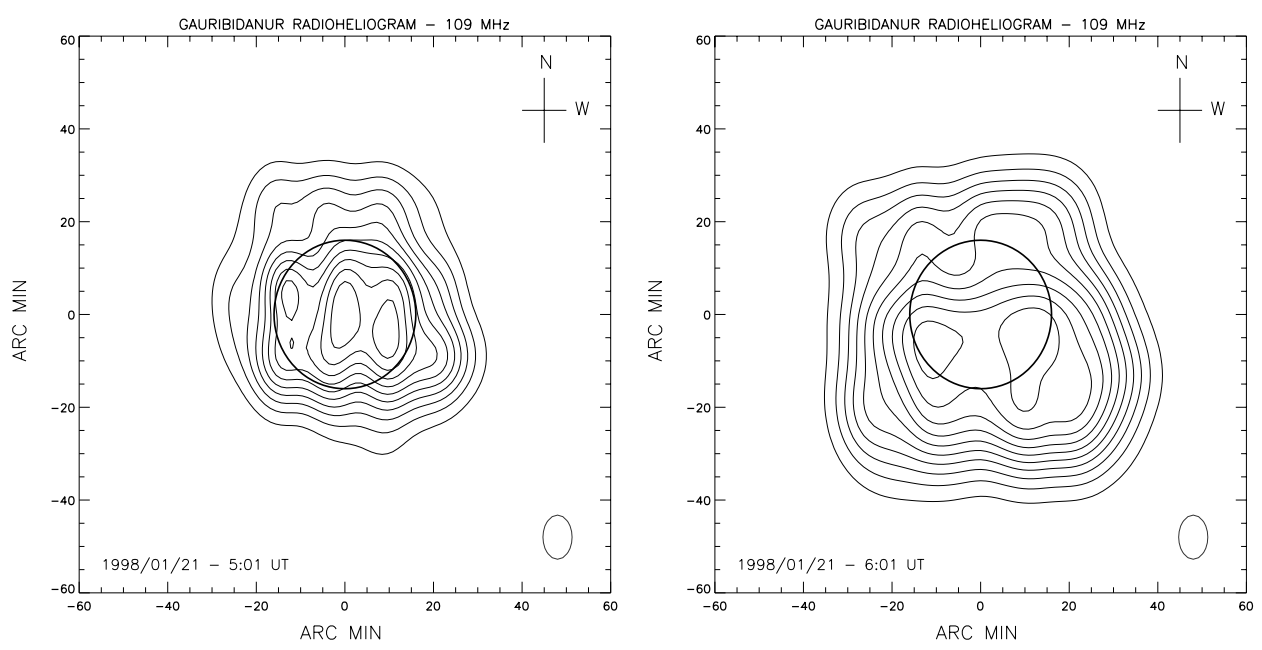

Figure 6. LEFT - Radioheliogram obtained with the GRH at $109 \mathrm{MHz}$ on January 21, 1998 at 05:01 UT. The peak brightness temperature is $\sim 1.21 \times 10^{6} \mathrm{~K}$ and the contour interval is $1 \times 10^{5}$ K. RIGHT - Same as in the left hand side, but obtained at 06:01 UT. The peak brightness temperature is $\sim 1 \times 10^{6} \mathrm{~K}$ and the contour interval is $1 \times 10^{5} \mathrm{~K}$.

in the plane of the sky was $361 \mathrm{~km} \mathrm{~s}^{-1}$. The extrapolated onset time of the event from the center of the Sun was 05:09:09 UT. The event was associated with a H-alpha filament disappearance from location (S57 E19) on the solar disk between 04:00 - 06:03 UT on that day (right hand side of Figure 5; Solar Geophysical Data, July 1998). Figure 6 shows the radioheliogram obtained with the GRH on January 21, 1998 around 05:01:06 \& 06:01:06 UT, respectively. The left hand side map in Figure 7 is the difference of the above two radioheliogram, i.e. 06:01:06 - 05:01:06 UT. One can notice enhanced radio emission at approximately the same location as the leading edge of the whitelight CME in the LASCO C2 difference image in Figure 5 (left hand side). The radio Sun was very 'quiet' and no non-thermal activity was reported, particularly during our observing period (Solar Geophysical Data, March 1998. This indicates that the emission seen in the left hand side map in Figure 7 is most likely due to thermal bremmstrahlung from the excess electrons (above the ambient) in the frontal loop of the whitelight CME, and is the radio counterpart of the latter.

\subsubsection{Analysis and Results}

The right hand side map in Figure 7 shows the brightness distribution obtained using the ray tracing technique described in section 2.2.1 for the observed radio map in the right hand side of Figure 6 . There is a striking similarity between them. The numbers s1-s16 indicate the location of the centroid of the discrete sources used in our ray tracing calculations [see Kathiravan \& Ramesh (2004) for further details]. A comparison of the two images in Figure 7 shows that there is a good correspondence between the location of the excess emission in the southern hemisphere of the latter and the sources $\mathrm{s} 8, \mathrm{~s} 9, \ldots, \mathrm{s} 13$ in the distribution obtained using ray tracing calculations. This suggests that the above set of discrete sources comprise the 'radio' CME. Its total mass $\left(M_{\text {radio }}\right)$ is given by,

$$
M_{\text {radio }}=m_{8}+m_{9}+m_{10}+m_{11}+m_{12}+m_{13}
$$

where $m_{8}, m_{9}, \ldots, m_{13}$ represent the mass of the structure $\mathrm{s} 8, \mathrm{~s} 9, \ldots, \mathrm{s} 13$ respectively. It was calculated by multiplying the volume of each one of them with the electron density at the location of their corresponding centroid. If $\vec{R}_{\text {radio }}$ indicates the position vector of 

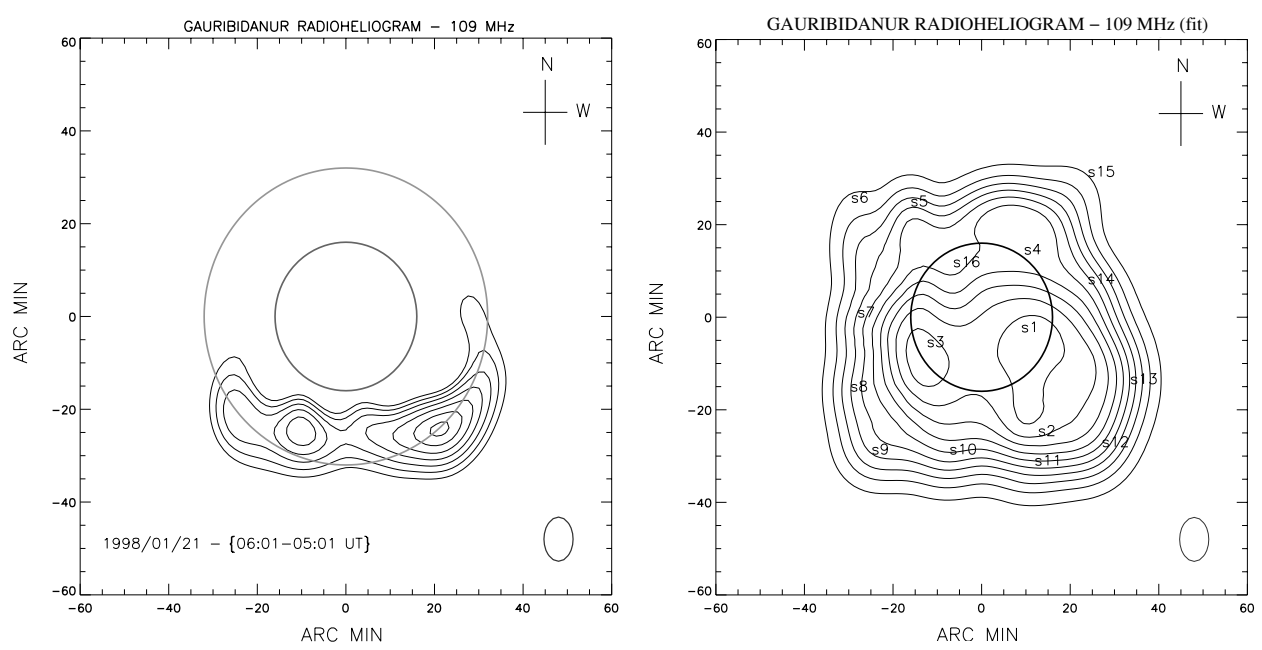

Figure 7. LEFT - Difference map obtained by subtracting the radioheliogram obtained at 05:01 UT from that at 06:01 UT in Figure 6. Contours with levels greater than 50\% of the peak value are only shown here. The enhanced radio emission in the southern hemisphere correlates well with the leading edge of the whitelight CME in Figure 5 (left hand side). The outer bigger circle in the map corresponds approximately to the occulter size of the LASCO C2 coronagraph. RIGHT - Radio brightness distribution of the Sun obtained through ray tracing calculations of the GRH data obtained at 06:01 UT (Figure 6). There is a good correspondence between the two distributions. The numbers s1-s16 indicate the location of the centroid of the discrete sources used in the ray tracing calculations.

the 'radio' CME, then we have

$$
\vec{R}_{\text {radio }}=\frac{1}{M_{\text {radio }}}\left[m_{8} \vec{r}_{8}+m_{9} \vec{r}_{9}+m_{10} \vec{r}_{10}+m_{11} \vec{r}_{11}+m_{12} \vec{r}_{12}+m_{13} \vec{r}_{13}\right]
$$

Here $\vec{r}_{8}, \vec{r}_{9}, \ldots, \vec{r}_{13}$ represent the position vector of $\mathrm{s} 8, \mathrm{~s} 9, \ldots, \mathrm{s} 13$, respectively. Substituting for the different values in equations $(2.5) \&(2.6)$ we get $\vec{R}_{\text {radio }}=1.16 \hat{x}+$ $0.52 \hat{y}-1.54 \hat{z}$. As pointed out earlier, the 'halo' CME of January 21, 1998 was closely associated with the disappearance of the $\mathrm{H} \alpha$ filament from the location (S57 E19) on the solar disk. Assuming the latter to be located on the solar surface, i.e. at a radial distance of $1 \mathrm{R}_{\odot}$ (in three-dimensional space) from the center of the Sun, we calculated its position vector as, $\vec{R}_{H \alpha}=0.51 \hat{x}-0.18 \hat{y}-0.84 \hat{z}$. According to the LASCO CME catalog, the liftoff time of the aforementioned 'halo' CME, projected to $1 \mathrm{R}_{\odot}$, is 05:41:17 UT. The above time was obtained by employing a linear fit to the LASCO C2/C3 height-time measurements of the leading edge of the whitelight CME in the plane of the sky, and is for the case where the CME is considered to travel at a constant speed. Note in the present case we have assumed that the source region of the CME on the solar disk is (S57 E19), i.e. the location of the $\mathrm{H} \alpha$ filament. This corresponds to a radial distance of $\approx$ $0.86 \mathrm{R}_{\odot}$ in the plane of the sky. We estimated the lift-off time of the CME from the above location by back-projecting its height-time curve, and it is 05:37:26 UT. This implies that the CME had travelled a distance of about $824,253 \mathrm{~km}\left[\right.$ i.e. $\sqrt{\left(\vec{R}_{\text {radio }}-\vec{R}_{H \alpha}\right)^{2}}$ in the three-dimensional space during the time interval 06:01:06 - 05:37:26 UT, i.e. at an average speed of $\approx 580 \mathrm{~km} \mathrm{~s}^{-1}$. Note that this value should be treated with caution since we have assumed that the CME was propagating at a constant speed. We also separately calculated its speed along the line of sight and in the plane of the sky, and they are $\approx 318 \& 485 \mathrm{~km} \mathrm{~s}^{-1}$, respectively. 


\section{Acknowledgements}

I thank Ch.V.Sastry and C.Kathiravan for their help in preparing this article. The staff of the Gauribidanur radio observatory are thanked for their help in data collection and maintenance of the antenna and receiver systems. The financial support provided by the International Astronomical Union and the Scientific Organizing Committee of the Symposium to attend the meeting and present this invited talk is acknowledged. The LASCO whitelight images presented are due to the kind courtesy of S.P.Plunkett, S.Yashiro and O.C.St.Cyr. The SOHO data are produced by a consortium of the Naval Research Laboratory (USA), Max-Planck-Institut fuer Aeronomie (Germany), Laboratoire d'Astronomie (France), and the University of Birmingham (UK). SOHO is a project of international cooperation between ESA and NASA. The CME catalog is generated and maintained by the Center for Solar Physics and Space Weather, the Catholic University of America in cooperation with the Naval Research Laboratory and NASA.

\section{References}

Aubier, M., Leblanc, Y. \& Boischot, A. 1971, Astron. Astrophys. 12, 435.

Bastian, T. S. \& Gary, D. E. 1997, J. Geophys. Res. 102(A7), 14031.

Bastian, T. S., Pick, M., Kerdraon, A., et al. 2001, ApJ 558, L65.

Brueckner, G. E., Howard, R. A., Koomen, M. J., et al. 1995, Solar Phys. 162, 357.

Dulk, G. A. 1980, in: M. R. Kundu \& T. E. Gergely (eds.), Radio Physics of the Sun, (Reidel: Dordrecht), IAU Symposium 86, p. 419.

Fleck, B., Domingo, V. \& Poland, V. (eds.), 1985, The SOHO Mission (Dordrect: Kluwer).

Fludra, A., Del Zenna, G., Alexander, D. \& Bromage, B. J. I. 1999, J. Geophys. Res. 104(A5), 9709.

Gopalswamy, N. \& Kundu, M. R. 1992, ApJ 390, L37.

Gopalswamy, N. 1999, in: T. Bastian, N. Gopalswamy \& K. Shibasaki (eds.), Solar Physics with radio observations, Nobeyama radio observatory report No. 479, p. 141

Gosling, J. T., McComas, D. J., Phillips, J. L. \& Bame, S. J. 1991, J. Geophys. Res. 96(A5), 7831.

Howard, R. A., Michels, D. J., Sheeley Jr. N. R. \& Koomen, M. J. 1982, ApJ 263, L101.

Kathiravan, C., Ramesh, R. \& Subramanian, K. R. 2002, ApJ 567, L93.

Kathiravan, C. \& Ramesh, R. 2004, ApJ 610, 532.

Newkirk, G. 1961, ApJ 133, 983.

Plunkett, S. P., Vourlidas, A., Simberová, S., et al. 2000, Solar Phys. 194, 371.

Ramesh, R., Subramanian, K. R., SundaraRajan, M. S. \& Sastry, Ch. V. 1998a, Solar Phys. $181,439$.

Ramesh, R. 1998b, Ph.D. Thesis Bangalore University.

Ramesh, R., Subramanian, K. R. and Sastry, Ch. V. 1999, Astron. Astrophys. Suppl. 139, 179.

Ramesh, R. 2000a, Solar Phys. 196, 213.

Ramesh, R. \& Sastry, Ch. V. 2000b, Astron. Astrophys. 358, 749.

Ramesh, R., Kathiravan, C. \& Sastry, Ch. V. 2001a, ApJ 548, L229.

Ramesh, R. \& Ebenezer, E. 2001b, ApJ 558, L141.

Ramesh, R. \& ShanmughaSundaram, G. A. 2001c, Solar Phys. 202, 355.

Ramesh, R., Kathiravan, C. and Sastry, Ch. V. 2003, ApJ 591, L163.

Sastry, Ch. V., Shevgaonkar, R. K. \& Ramanuja, M. N. 1983, Solar Phys. 87, 391.

Sheridan, K. V., Jackson, B. V., McLean, D. J. \& Dulk, G. A. 1978, Proc. Astron. Soc. Australia $3(4), 249$.

Smerd, S. F. 1950, Aust. J. Sci. Res. A3, 34.

Solar Geophysical Data 643 (Part I), March 1998.

Solar Geophysical Data 646 (Part I), June 1998.

Solar Geophysical Data 647 (Part II), July 1998.

Solar Geophysical Data 648 (Part I), August 1998. 
Solar Geophysical Data 677 (Part I), January 2001.

Vourlidas, A., Subramanian, P., Dere, K. P. \& Howard, R. A.2000 ApJ 534, 456.

\section{Discussion}

Bothmer : Does L 160.000 Mm for June 2, 98 event correspond roughly to length of the arcade of loops ? $\mathrm{L} \sim 160.000 \mathrm{Mm} \approx 20^{\circ}$ in heliolog.

RAMESH: The June 2, 1998 radioheliogram presented was observed about 10 min prior to the appearance of the associated CME in the LASCO field of view. So, it is possible that the estimated line of sight depth of the discrete radio structure(s) would be the location of the CME correspond to a pre-event coronal arcade system.

FILIPPOv : You showed the trajectory of a partial halo CME that was not the straight line but the curved one. Is it real non-radial material motion or the result of brightness redistribution within radially spreading CME body?

RAMESH: We followed the centroid of the discrete radio structure (whose position correlated well with the leading edge of the white light CME in the LASCO FoV) to obtain the trajectory of the 'radio' CME. The estimated peak brightness temperature of the structure did not change much during our observing interval. This indicates that the trajectory is mostly real.

Grechnev : 1 . Have you measured the temporal variation of the acceleration?

2. Can you comment on the brightness temperatures you've shown? They seem to be rather low; is this due to calibration techniques?

RAMESH: 1. Yes, we have sufficient time resolution but I do not have a picture here.

2. The low brightness temperature reported are not due to any calibration errors. Note that the radio brightness temperature due to a CME is always less than $10^{6} \mathrm{~K}$. This is mostly because the CME is optically thin. Also it should be kept in mind that we have refraction effects off the limb. This also could lead to a reduced brightness temperature.

JINGXIU WANG: What is the spatial resolution of your radioheliogram, if the resolution is good enough to study the CME initiation process?

RAMESH: The spatial resolution of the Gauribidanur radioheliograph is approximately $5^{\prime}$. The instrument is better suited to study the global coronal changes associated with the onset of a CME. 